\title{
Four-wave mixing in integrated ring resonators integrated with 2D graphene oxide
}

\author{
Jiayang Wu, ${ }^{1}$ Yunyi Yang, ${ }^{1}$ Y uning Zhang, ${ }^{1}$ Yang Qu, ${ }^{1}$ Linnan Jia, ${ }^{1}$ Xingyuan $\mathrm{Xu},{ }^{1}$ \\ Sai T. Chu, ${ }^{2}$ Brent E. Little, ${ }^{3}$ Roberto Morandotti, ${ }^{4,5}$ Baohua Jia, ${ }^{1}$ and David J. Moss ${ }^{1 *}$ \\ ${ }^{I}$ Centre for Micro-Photonics, Swinburne University of Technology, Hawthorn, Victoria 3122, Australia \\ ${ }^{2}$ Department of Physics, City University of Hong Kong, 83 Tat Chee Avenue, Hong Kong, China. \\ ${ }^{3} X i$ 'an Institute of Optics and Precision Mechanics Precision Mechanics, Chinese Academy of Science, Xi'an, China. \\ ${ }^{4}$ INRS-Énergie, Matériaux et Télécommunications, 1650 Boulevard Lionel-Boulet, Varennes, Québec, J3X 1S2, Canada. \\ ${ }^{5}$ Institute of Fundamental and Frontier Sciences, University of Electronic Science and Technology of China, Chengdu 610054, China. \\ Email:dmoss@swin.edu.au
}

\begin{abstract}
We experimentally demonstrate enhanced four-wave mixing in micro-ring resonators (MRRs) integrated with graphene oxide films. We achieve up to 7.6- $\mathrm{dB}$ enhancement in conversion efficiency for a uniformly coated MRR and $\sim 10.3-\mathrm{dB}$ for a patterned device.
\end{abstract}

\section{Introduction}

Four-wave mixing (FWM), as a fundamental third-order $\left(\chi^{(3)}\right)$ nonlinear optical process [1], has been widely used for all-optical signal generation and processing, such as wavelength conversion, optical comb generation, quantum entanglement, optical sampling, and many other [2-5]. Integrated micro-ring resonators (MRRs), with strong light confinement in compact micro-scale resonant cavities, are key building blocks for photonic integrated circuits [6-8]. Compared with FWM in integrated waveguides, FWM in integrated MRRs can provide dramatically enhanced conversion efficiencies (CE) due to resonance enhancement, thus significantly reducing the power requirements.

Although silicon offers many benefits as a linear optical platform, for nonlinear optics it suffers from strong twophoton absorption (TPA) in the telecommunications band, which greatly limits the FWM performance [1]. Other integrated platforms such as silicon nitride and high index doped silica glass, though have a much weaker TPA, still face limitations in terms of FWM efficiency since their Kerr nonlinearity $\left(n_{2}\right)$ is over an order of magnitude smaller than that of silicon $[9,10]$. In this paper, we demonstrate enhanced FWM in MRRs integrated with layered graphene oxide (GO) films. Both uniformly coated and patterned GO films are integrated on CMOS-compatible doped silica MRRs using a large-area, transfer-free, layer-by-layer GO coating method together with photolithography and liftoff processes, yielding precise control of the film thickness, placement, and coating length. Owing to the strong lightmatter interaction in the MRRs incorporating highly nonlinear GO films, the FWM efficiency in the hybrid MRRs is significantly improved. We achieve up to 7.6-dB enhancement in the FWM CE for an MRR uniformly coated with 1 layer of GO and $\sim 10.3-\mathrm{dB}$ for a device patterned with 50 layers of GO. These results confirm the high nonlinear optical performance of integrated photonic resonators incorporated with layered GO films.

\section{Four-wave mixing in GO-coated MRRs}

Figure 1(a) shows a schematic of an integrated MRR incorporating a GO film. The MRR was fabricated on a high index doped silica glass platform using CMOS compatible fabrication processes [11, 12]. Chemical mechanical polishing was used as the last step to remove the upper cladding, so as to enable GO film coating on the top surface of the MRR. The coating of layered GO films was achieved via a solution-based method that yields transfer-free [13], layer-by-layer GO film deposition [14]. Based on this GO coating method, we achieved GO patterning on integrated photonic devices via photolithography and lift-off processes [15]. Figure 1(b) shows microscopic images of an

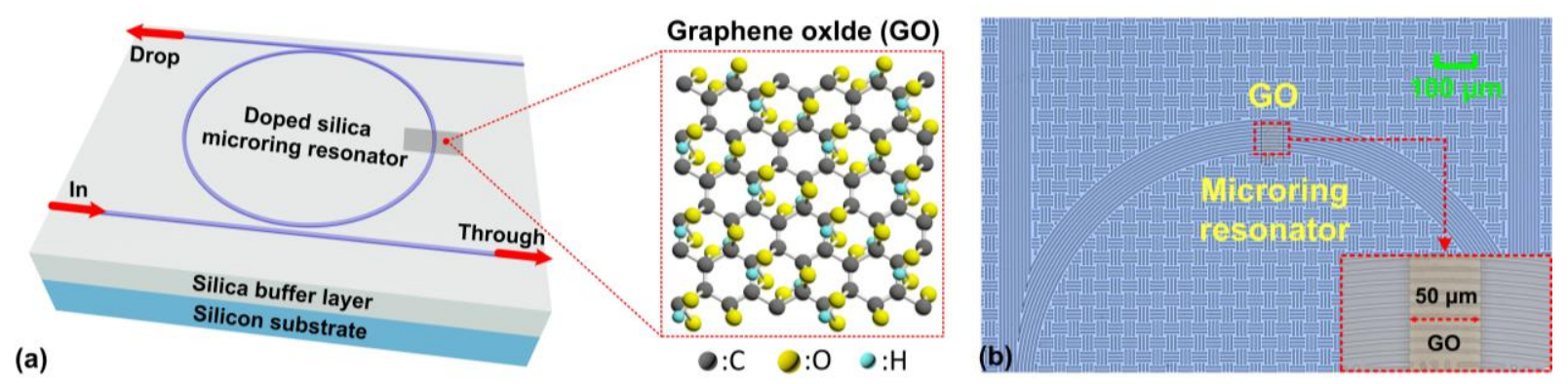

Fig. 1. (a) Schematic illustration of GO-coated integrated MRR. Inset shows schematic atomic structure of GO. (b) Microscopic image of an integrated MRR patterned with 50 layers of GO. Inset shows zoom-in view of the patterned GO film. 
integrated MRR patterned with 50 layers of $\mathrm{GO}(\sim 50 \mu \mathrm{m}$ pattern length $)$. The GO film thickness shows a nearly linear relationship with the layer number, with a thickness of $\sim 2.25 \mathrm{~nm}$ on average for each layer.
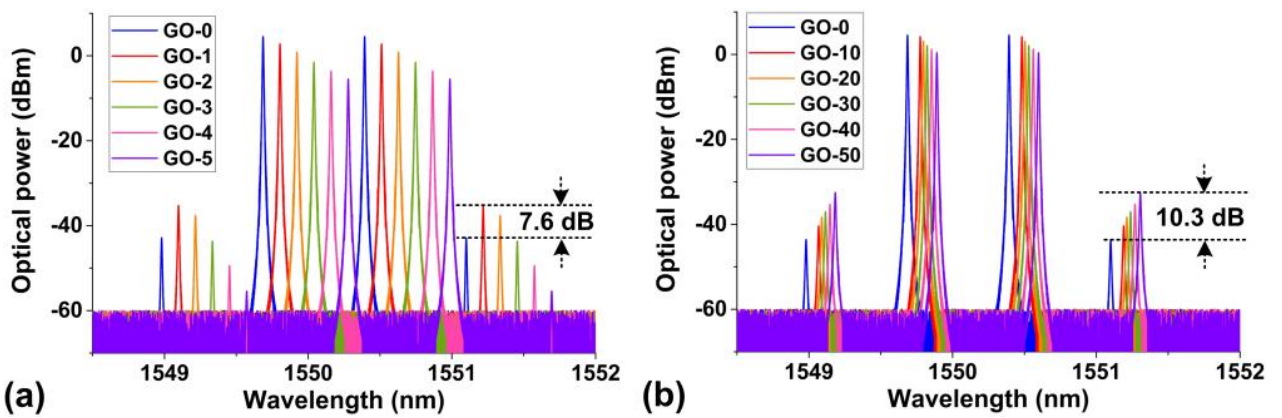

Fig. 2. Optical spectra of FWM at a pump power of $22 \mathrm{dBm}$ for the MRRs with (a) 1-5 layers of uniformly coated and (b) 10-50 layers of patterned GO films, respectively. The results for uncoated MRR (GO-0) are also shown for comparison.

Figure 2(a) shows the FWM spectra of the MRRs uniformly coated with 1-5 layers of GO, together with the FWM spectrum of the uncoated MRR. For comparison, we kept the same pump power of $\sim 22 \mathrm{dBm}$ coupled into the MRRs. As compared with the uncoated MRR, the GO-coated MRRs had an additional insertion loss, while the MRRs with 1 and 2 layers of GO clearly show enhanced idler output powers. The CE of the MRR without GO and with 1 layer of GO were $\sim-48.4 \mathrm{~dB}$ and $\sim-40.8 \mathrm{~dB}$, respectively, corresponding to a $\mathrm{CE}$ enhancement of $7.6 \mathrm{~dB}$ for the GO-coated MRR. Figure 2(b) shows the FWM spectra of the MRRs with 10-50 layers of patterned GO. The GO coating length was $\sim 50 \mu \mathrm{m}$ and the pump power $(22 \mathrm{dBm})$ was the same as that in Fig. 2(a). The results for all the tested GO layer numbers show enhanced idler output powers. In particular, there is a maximum CE enhancement of $\sim 10.3 \mathrm{~dB}$ for the MRR patterned with 50 layers of GO. Figures 3(a)-(c) show the FWM spectra versus $\Delta \lambda$ (wavelength spacing between pump and signal) for the uncoated MRR, the uniformly coated MRR with 1 layer of GO, and the patterned MRR with 50 layers of GO, respectively. The measured CE versus $\Delta \lambda$ is depicted in Figure 3(d) where we see that, for all three MRRs, the CE only shows a slight decrease with $\Delta \lambda$, reflecting the low dispersion of the doped silica MRR and the GO-coated MRRs, thus enabling effective phase matching for broadband FWM.

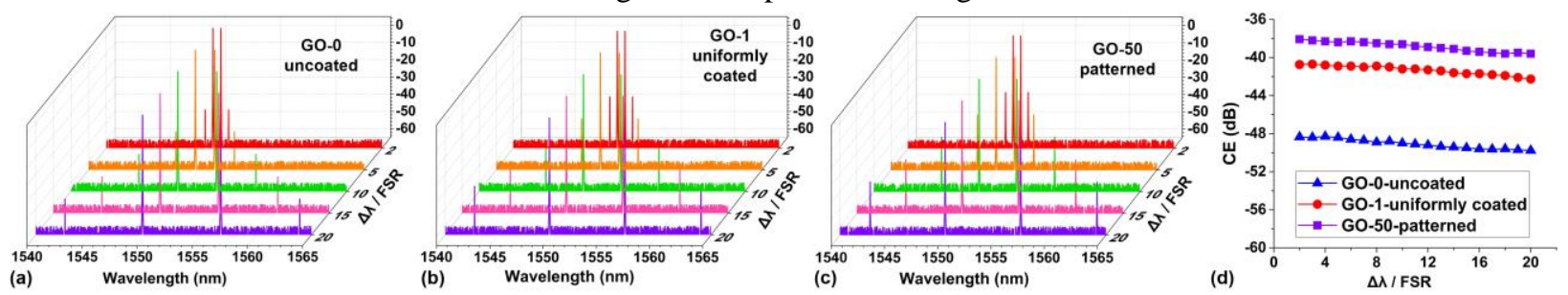

Fig. 3. (a)-(c) Optical spectra of FWM at different resonant wavelengths for the uncoated MRR, the MRR uniformly coated with 1 layer of GO, and the MRR patterned with 50 layers of GO, respectively. $\Delta \lambda$ and FSR represent the wavelength spacing between pump and signal and the free spectral ranges of the MRRs, respectively. (d) Measured CE versus $\Delta \lambda / F S R$ for the MRRs in (a) - (c). The pump power in (a) $-(\mathrm{d})$ was $22 \mathrm{dBm}$.

\section{Conclusion}

We demonstrate enhanced FWM in MRRs integrated with layered GO films. We perform FWM measurements for MRRs uniformly coated and patterned with GO films, achieving up to 7.6-dB and $~ 10.3-\mathrm{dB}$ enhancement in the FWM CE for the MRRs uniformly coated with 1 layer of GO and patterned with 50 layers of GO, respectively.

\section{References}

[1] J. Leuthold, C. Koos, and W. Freude, "Nonlinear silicon photonics," Nat. Photon., 4 (8), 535, 2010.

[2] J. Wu et.al., "RF photonics: an optical microcombs' perspective," J. Sel. Top. Quantum Electron., 24 (4), $1,2018$.

[3] M. Kues et.al., "On-chip generation of high-dimensional entangled quantum states and their coherent control," Nature, 546 (7660), $622,2017$.

[4] A. Pasquazi et.al., "All-Optical wavelength conversion in an integrated ring resonator”, Opt. Exp. 18 (4) $3858,2010$.

[5] X. Xu et.al., "Broadband RF channelizer based on an integrated optical frequency Kerr comb source," J. Lightw. Technol., 36 (19), $4519,2018$.

[6] J. Wu et.al., "Micro-ring resonator quality factor enhancement via an integrated Fabry-Perot cavity" APL Photonics, 2 (5), 056103, 2017.

[7] M. Xu et.al., "Push-pull optical nonreciprocal transmission in cascaded silicon microring resonators," Photon. J., 5 (1), $2200307,2013$.

[8] J. Wu et.al., "Nested configuration of silicon microring resonator with multiple coupling regimes," Photon. Technol. Lett., 25 (6), $583,2013$.

[9] D. Moss et.al., "New CMOS-compatible platforms based on silicon nitride and Hydex for nonlinear optics," Nat. Photon., 7 (8), $597,2013$.

[10] X. Xu et.al., "Advanced RF and microwave functions based on an integrated optical frequency comb source," Opt. Exp., 26 (3), $2569,2018$.

[11] X. Xu et.al., "Continuously tunable orthogonally polarized RF optical single sideband generator based on micro-ring resonators," Journal of Opt., 20 (11), 115701, 2018.

[12] H. Bao et al., "Laser cavity-soliton microcombs," Nat. Photon., 13 (6), 384, 2019.

[13] Y. Yang et.al., "Bottom-up fabrication of graphene on silicon/silica substrate via a facile soft-hard template approach,” Sci. Rep., 5, 1, 2015

[14] Y. Yang et.al., "Enhanced four-wave mixing in waveguides integrated with graphene oxide," APL Photonics, 3 (12), $120803,2018$.

[15] J. Wu et.al., "Graphene oxide waveguide and micro-ring resonator polarizers," Laser Photonics Rev., 13 (9), 1900056, 2019. 